\title{
Perfil de los pacientes con trastorno adaptativo que acuden a las farmacias comunitarias españolas
}

\author{
Jesús C. Gómez Martínez', Fernanda López-Laguna Guerrero², \\ José Ferragud Masià ${ }^{3}$, Montserrat Abadías Guasch ${ }^{4}$ \\ 1. Farmacéutico comunitario, Barcelona. 2. Farmacéutico comunitario, Madrid. 3. Médico psiquiatra. Centro Salud Mental Malvarrosa, Valencia. \\ 4. Médico farmacólogo. Medical Department and Health Innovation, Esteve, Barcelona.
}

\section{PALABRAS CLAVE}

depresión, estado de ánimo, trastorno adaptativo, farmacia comunitaria

\section{ABREVIATURAS}

DSM-IV: Manual Diagnóstico y Estadístico de Trastornos Mentales. OMS: Organización Mundial de la Salud. SF-12: Short-Form 12. EQ-5D: European Quality of Life - 5 Dimensions. DE: desviación estándar. Q: cuartil. ODIN: Outcome of Depression International Network Project. 5-HT: serotonina. IC: intervalo de confianza.

\section{KEYWORDS}

depression, mood, adjustment disorder, community pharmacy

\section{RESUMEN}

Objetivo: El objetivo principal era conocer los perfiles de los sujetos que cumplían los criterios diagnósticos de trastorno adaptativo en la consulta habitual de las farmacias comunitarias españolas.

Material y métodos: Estudio transversal de ámbito nacional sobre una muestra de 1.512 sujetos. Se evaluaron las características del perfil socio-demográfico y clínico, así como del estado de ánimo y de salud, recogidos mediante entrevista con el farmacéutico. El estado de ánimo se valoró mediante una escala de expresiones faciales con gradación del 1 (risa) al 7 (llanto). El estado de salud se valoró mediante preguntas sobre movilidad, cuidado personal, dolor y malestar, sueño y energía, ánimo, habilidades interpersonales y cognición. La duración total de la fase experimental fue de 7 meses. Se utilizó básicamente estadística descriptiva.

Resultados: La población de sujetos estudiados que cumplían criterios diagnósticos de trastorno adaptativo tenía una edad media de $43,1 \pm 14,8$ años, siendo un $63,0 \%$ mujeres. El $61,8 \%$ de los sujetos consultó por estado de ánimo deprimido con un tiempo de evolución de la mayor parte del día en el $60,8 \%$ de ellos y durante más de 2 semanas en el 79,0\% de ellos. El 37,1\% de los sujetos valoró su estado de ánimo como triste (gradación 5-7). El 38,1\% de sujetos presentaba estado de ánimo deprimido y ansiedad. El 12,0\% de los sujetos presentaba síntomas depresivos subclínicos. Se observó relación significativa $(p<0,001)$ entre problemas emocionales y salud física.

Conclusión: Los síntomas más frecuentes de los pacientes que refieren sintomatología de trastorno adaptivo en este estudio son consulta frecuente en la farmacia comunitaria, lo que podría sugerir que los farmacéuticos comunitarios podrían ayudar con su intervención farmacéutica en la detección precoz y abordaje de esta patología.

\section{Profile of patients with adaptive disorder visiting Spanish community pharmacies}

\section{ABSTRACT}

Objective: The main objective was to know the profiles of subjects meeting the diagnostic criteria for adjustment disorder, in the usual consultation of the Spanish community pharmacies.

Material and Methods: Cross-sectional study at national level over a sample of 1512 subjects. The socio-demographic and clinical profile characteristics were evaluated, as well as mood and health status, obtained by interview with the pharmacist. Mood was assessed by a facial expression scale graded from 1 (laughter) to 7 (tears). Health status was assessed with questions on mobility, self-care, pain and discomfort, sleep and energy, mood, interpersonal skills and cognition. The total length of the experimental phase was of 7 months. Descriptive statistics was mainly used.

Results: The studied subject population meeting the diagnostic criteria for adjustment disorder had a mean age of $43.1 \pm 14.8$ years, being $63.0 \%$ women. The $61.8 \%$ of subjects consulted due to depressed mood most of the day in the $60.8 \%$ of the subjects and during more than 2 weeks in the $79.0 \%$ of subjects. The $37.1 \%$ of subjects assessed their mood as sad (grading 5-7). The $38.1 \%$ of subjects presented depressed mood and anxiety. The $12.0 \%$ of subjects presented subclinical depressive symptoms. A significant relationship was found $(\mathrm{p}<0.001)$ between emotional troubles and physical health.

Conclusion: Most frequent symptoms from patients referring symptoms of adjustment disorder in this study are cause of frequent consultation in the community pharmacy, which would suggest that the community pharmacists would help in the early detection and approach of this pathology with their intervention. 


\section{Introducción}

Los trastornos adaptativos se caracterizan por una reacción de desajuste que puede presentarse con sintomas emocionales y/o de comportamiento en respuesta a uno o varios factores estresantes psicosociales identificables que ocurren en un corto espacio de tiempo desde el inicio del factor de estrés, y su diagnóstico se realiza de acuerdo con los criterios diagnósticos de la cuarta edición del Manual Diagnóstico y Estadístico de Trastornos Mentales (DSM-IV) $(1,2)$.

De esta forma, el trastorno adaptativo constituye habitualmente un diagnóstico de exclusión y está en el límite entre la categoría de enfermedad y de problema, y por tanto sujeto a un considerable vaivén ideológico (3).

En cuanto a las características de las personas que padecen un trastorno adaptativo, algunos estudios señalan que suelen ser más jóvenes, independientes económicamente y que suelen convivir con otras personas, en comparación con las que presentan un diagnóstico psiquiátrico mayor $(3,4)$.

Dada la ausencia de estudios epidemiológicos respecto al trastorno adaptativo se hace necesario la identificación del perfil socio-demográfico y clínico de los sujetos que cumplen con los criterios de este trastorno con el fin de conocer los diferentes patrones de este trastorno en España, particularmente en los subtipos más frecuentes (con estado de ánimo depresivo, mixto con ansiedad y con estado de ánimo depresivo).

Por otro lado, el trastorno adaptativo se encuentra en el límite entre la normalidad y la patología, y se caracteriza por síntomas que pueden ocurrir sin necesidad de que el sujeto detecte que puede sufrir una patología, con fatiga y deterioro en las actividades cotidianas. En muchas ocasiones, el sujeto puede que no acuda a su médico de familia a consultar estos sintomas y demande ayuda en su farmacia habitual, solicitando consejo y apoyo para esta situación.

El objetivo principal del estudio era conocer el perfil de los sujetos que cumplían los criterios diagnósticos de trastorno adaptativo en las farmacias comunitarias españolas. Los objetivos secundarios eran conocer en esta población de estudio la frecuencia de sujetos con estado de ánimo deprimido, estado de ánimo deprimido $\mathrm{y}$ ansioso, y conocer la frecuencia de sintomas depresivos subclínicos.

\section{Material y métodos}

Diseño del estudio y selección de sujetos

Estudio observacional no postautorización, transversal, multicéntrico y de ámbito nacional, realizado en sujetos que cumplían los criterios de DSM-IV (1) de trastorno adaptativo, con la colaboración de 300 farmacéuticos de farmacias comunitarias distribuidas por todo el territorio nacional. Para su inclusión en el estudio los sujetos participantes debían tener además edad igual o superior a 18 años, aparición de síntomas emocionales o de comportamiento en respuesta a un estresante identificable (dentro de los 3 meses siguientes a la presencia del estresante), los síntomas o comportamientos debían expresarse clínicamente como malestar mayor de lo esperable en respuesta al estresante y deterioro significativo de la actividad social o laboral o académica, la alteración relacionada con el estrés no debía cumplir los criterios para otro trastorno específico ni constituir una simple exacerbación de un trastorno preexistente. Constituian criterios de exclusión los síntomas que respondian a una reacción de duelo, los sintomas que persistieron más de 6 meses una vez cesado el estresante, la existencia de una patología o tratamiento que interfiriera en la adecuada valoración de los criterios anteriores, y deterioro cognitivo suficiente para que impidiera la comprensión de lo redactado en el consentimiento informado.

El estudio se realizó siguiendo las guías de Buenas Prácticas Clínicas de la Conferencia Internacional de Armonización (5) y los requerimientos expresados en la Declaración de Helsinki (revisión de Seúl, Octubre de 2008), así como la legislación vigente en España según lo dispuesto en la orden ministerial SAS/2470/2009 en relación con la realización de estudios observacionales. El estudio fue aprobado por el Comité Ético de Investigación Clínica del Hospital Clínic de Barcelona, y se ajustó a lo dispuesto en la Ley Orgánica 15/1999, de 13 de diciembre, de protección de datos de carácter personal. Todos los sujetos participantes en el estudio firmaron el correspondiente formulario de consentimiento informado antes de iniciar su participación.
Variables del estudio

El estudio planteó una recogida transversal de datos, de manera que la obtención de datos se realizó en una única visita del sujeto a la farmacia comunitaria, siendo la fuente de información en todos los casos el propio sujeto participante mediante entrevista con el farmacéutico. Entrevista estructurada para efectuar la selección de los sujetos según un algoritmo preestablecido, obtener el consentimiento informado por escrito y realizar la evaluación del estado de ánimo y salud del sujeto de acuerdo a los criterios preestablecidos (véase anexo, guía específica del estudio). Dicha entrevista se realizó a los cinco primeros sujetos que acudian a cada una de las farmacias comunitarias demandando ayuda (farmacológica y/o suplementos y/o consejo), por referir síntomas emocionales o de comportamiento que podían corresponderse con un trastorno adaptativo. Cada farmacia comunitaria debía incluir al menos cinco sujetos. Todos los datos se recogieron en un cuaderno de recogida de datos estandarizado, entre los meses de junio y diciembre de 2012 .

Las variables a analizar incluían las características del perfil socio-demográfico, clínico, del estado de ánimo y de salud. El estado de ánimo se valoró mediante una escala de expresiones faciales con gradación del 1 (risa) al 7 (llanto) pasando por rostros intermedios con diferente grado de felicidad o tristeza (6).

El estado de salud presente en los últimos 30 días se valoró mediante una serie de preguntas basadas en los dominios de salud y las preguntas incluidas en la Encuesta Mundial de Salud de la OMS, y que se utilizan habitualmente en los cuestionarios como el Short-Form 12 (SF-12) (7) o el EuroQol (EQ-5D), (8) agrupadas en 7 dominios de salud: 1) movilidad - dificultades para moverse en su entorno y para llevar a cabo actividades de cierto vigor; 2) cuidado personal - dificultades en el autocuidado y mantenimiento de la apariencia física; 3) dolor y malestar - dolores corporales y malestar corporal general; 4) sueño y energía - dificultad para dormir y sentirse descansado y recuperado; 5) ánimo - sentimientos de tristeza, desánimo, preocupación y ansiedad; 
6) habilidades interpersonales - dificultad para las relaciones personales y la participación en la comunidad, y para manejar conflictos y tensiones; y 7) cognición - dificultad para concentrarse y recordar cosas y dificultad para aprender una nueva tarea.

También se tuvieron en cuenta los sintomas descritos motivo de consulta y el tiempo de evolución. Las variables secundarias fueron la proporción de sujetos con estado de ánimo deprimido, con estado de ánimo deprimido y ansiedad, y la presencia de sintomas depresivos subclínicos en la población estudiada (estado de ánimo deprimido, pérdida de interés y fatiga).

\section{Análisis estadístico}

Las variables cuantitativas se describieron con medidas de centralización y de dispersión (media, desviación estándar [DE], mediana, primer y tercer cuartil $\left[\mathrm{Q}_{1} \mathrm{y} \mathrm{Q}_{3}\right]$, mínimo y máximo), y los correspondientes intervalos de confianza al 95\% (IC 95\%). Los resultados se expresaron como media \pm DE o mediana (rango). Las variables cualitativas se describieron mediante las frecuencias absolutas y relativas. En el análisis de tablas de contingencia se utilizó el test de chi-cuadrado (o el test exacto de Fisher según procediera). Todos los análisis se realizaron con el paquete estadístico SPSS $^{\circledR}$ V17.

\section{Resultados}

De los 1.606 sujetos inicialmente incluidos, se excluyeron 94 (5,9\%) por no cumplir los criterios de inclusión/exclusión, por lo que el número de pacientes evaluables fue de 1.512 (94,1\%).

\section{Datos sociodemográficos}

En la tabla 1 constan los datos socio-demográficos detallados. La edad media de los sujetos participantes en el estudio era de $43,1 \pm 14,8$ años, con un 63,0\% de mujeres. El 38,1\% de los sujetos tenía estudios superiores universitarios, y el 62,2\% de los sujetos era laboralmente activo.

Los factores estresantes mayoritarios fueron dificultades económicas en el 39,9\%, y problemas familiares en el $32,9 \%$.
Tabla 1 Caracteristicas sociodemográficas

\begin{tabular}{|c|c|c|c|}
\hline & $n$ & Media $\pm \mathrm{DE} \%$ & IC $95 \%$ \\
\hline Edad (años) & 1.494 & $43,1 \pm 14,8$ & $42,4-43,9$ \\
\hline Sexo & 1.496 & & \\
\hline - Hombre & 553 & $37,0 \%$ & $34,5-39,5$ \\
\hline - Mujer & 943 & $63,0 \%$ & $60,5-65,5$ \\
\hline \multicolumn{4}{|l|}{ Raza } \\
\hline - Caucásica & 1.386 & $95,5 \%$ & $94,3-96,5$ \\
\hline - Otra & 66 & $4,6 \%$ & - \\
\hline \multicolumn{4}{|l|}{ Nacionalidad } \\
\hline - Española & 1.430 & $96,6 \%$ & $95,5-97,4$ \\
\hline - Otra & 51 & $3,4 \%$ & $2,6-4,5$ \\
\hline \multicolumn{4}{|l|}{ Estado civil } \\
\hline - Casado & 755 & $50,0 \%$ & $47,4-52,5$ \\
\hline - Soltero & 515 & $34,1 \%$ & $31,7-36,5$ \\
\hline - Divorciado & 95 & $6,3 \%$ & $5,1-7,6$ \\
\hline - Separado & 76 & $5,0 \%$ & $4,0-6,3$ \\
\hline - Otro & 70 & $4,6 \%$ & $3,6-5,8$ \\
\hline \multicolumn{4}{|l|}{ Situación de convivencia } \\
\hline - Familiar & 871 & $57,6 \%$ & $55,1-60,1$ \\
\hline - En pareja & 365 & $24,1 \%$ & $22,0-26,4$ \\
\hline - Solo & 240 & $15,9 \%$ & $14,1-17,8$ \\
\hline - Otros & 6 & $0,4 \%$ & $0,1-0,9$ \\
\hline \multicolumn{4}{|l|}{ Nivel de estudios } \\
\hline - Superiores & 576 & $38,1 \%$ & $25,7-40,6$ \\
\hline - Secundarios & 547 & $36,2 \%$ & $33,8-38,7$ \\
\hline - Primarios & 309 & $20,5 \%$ & $18,4-22,6$ \\
\hline - Sin estudios & 72 & $4,8 \%$ & $3,7-6,0$ \\
\hline - Otros estudios & 7 & $0,5 \%$ & $02,-1,0$ \\
\hline \multicolumn{4}{|l|}{ Situación laboral } \\
\hline - Activo & 941 & $62,2 \%$ & $59,7-64,9$ \\
\hline - Paro & 178 & $11,8 \%$ & $10,2-13,5$ \\
\hline - Jubilado & 135 & $8,9 \%$ & $7,5-10,5$ \\
\hline - Ama de casa & 114 & $7,5 \%$ & $6,3-9,0$ \\
\hline - Estudiante & 83 & $5,5 \%$ & $4,4-6,8$ \\
\hline - Baja laboral & 30 & $2,0 \%$ & $1,3-2,8$ \\
\hline - Incapacidad laboral o invalidez permanente & 18 & $1,2 \%$ & $0,7-1,9$ \\
\hline - Otros & 13 & $0,9 \%$ & - \\
\hline \multicolumn{4}{|l|}{ Factores estresantes } \\
\hline - Dificultades económicas & 604 & $39,9 \%$ & $37,5-42,5$ \\
\hline - Problemas familiares & 497 & $32,9 \%$ & $30,5-42,5$ \\
\hline - Fracaso en objetivos profesionales (o académicos) & 271 & $17,9 \%$ & $16,0-20,0$ \\
\hline - Pérdida de empleo & 269 & $17,8 \%$ & $15,9-19,8$ \\
\hline - Otros & 964 & $63,8 \%$ & - \\
\hline
\end{tabular}

DE: desviación estándar; IC: intervalo de confianza; n: número de sujetos; \%: porcentaje. 


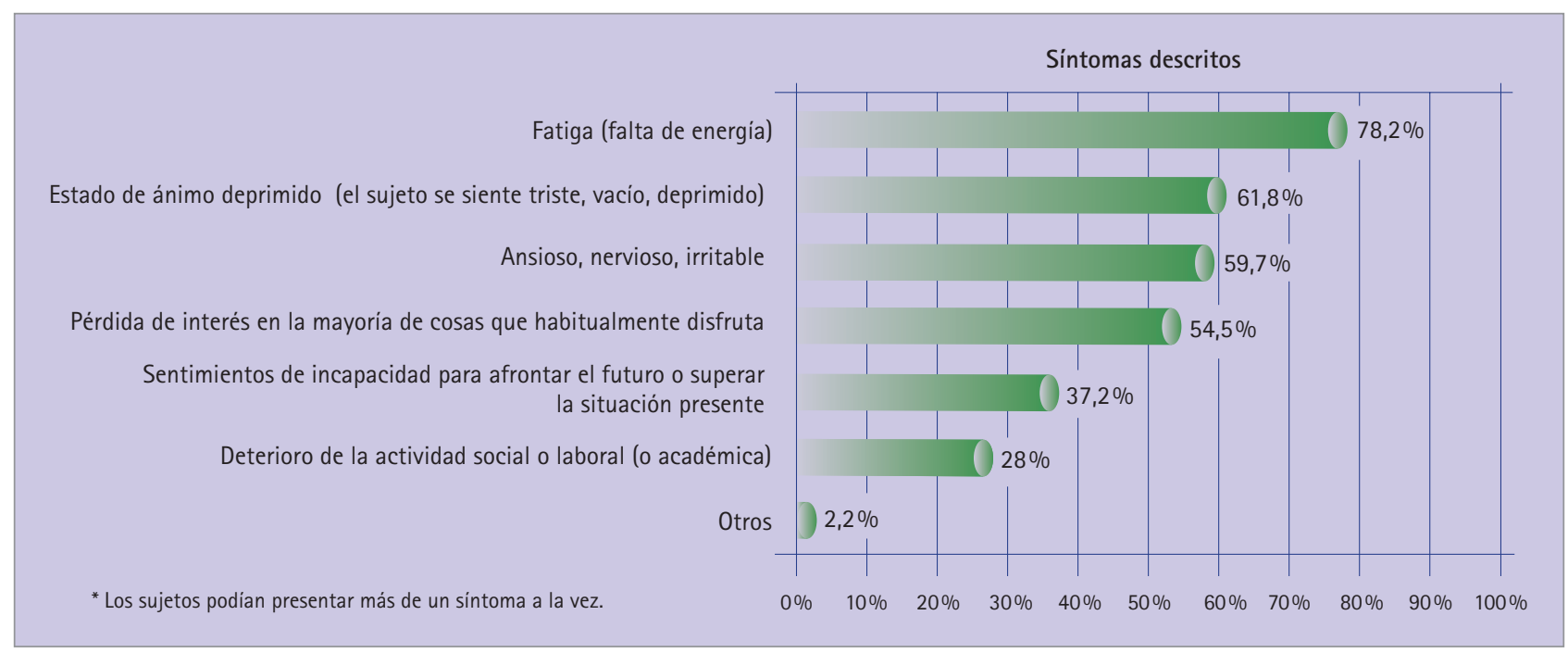

Figura 1 Sintomas descritos motivo de la consulta

\section{Datos relacionados}

con el trastorno adaptativo

Los principales síntomas descritos como motivo de la consulta fueron en el 78,2\% de los sujetos fatiga/falta de energía, y en el $61,8 \%$ estado de ánimo deprimido (el sujeto se sentía triste, vacío, deprimido) (figura 1). El tiempo de evolución en el caso de fatiga/falta de energía fue de la mayor parte del día en el 78,4\% de sujetos y durante más de 2 semanas en el $78,1 \%$. En el caso de estado de ánimo deprimido, fue de la mayor parte del día en el 60,8\% de sujetos y durante más de 2 semanas en el 79,0\%. En caso de pérdida de interés en la mayoría de cosas con las que habitualmente disfrutaba, fue de la mayor parte del día en el 69,9\% de sujetos y durante más de 2 semanas en el $72,5 \%$. Globalmente, el tiempo medio de evolución del motivo de la consulta fue de $1,9 \pm 1,4$ meses.

Factores clínicos, enfermedades relevantes pasadas y activas

Las alteraciones más frecuentes de los sujetos participantes fueron hipertensión en el 18,8\% de sujetos, artritis/ artrosis en el 14,9\% de sujetos, úlceras gastrointestinales en el 7,5\% de sujetos, y depresión u otros trastornos afectivos en el 7,0\% de los sujetos. Siendo por depresión en el 14,2\% de sujetos, ansiedad en el 13,2\% de sujetos e insomnio en el 5,7\% de los sujetos.

\section{Estado de ánimo y estado de salud}

Al evaluar el estado de ánimo en la escala de expresiones faciales con

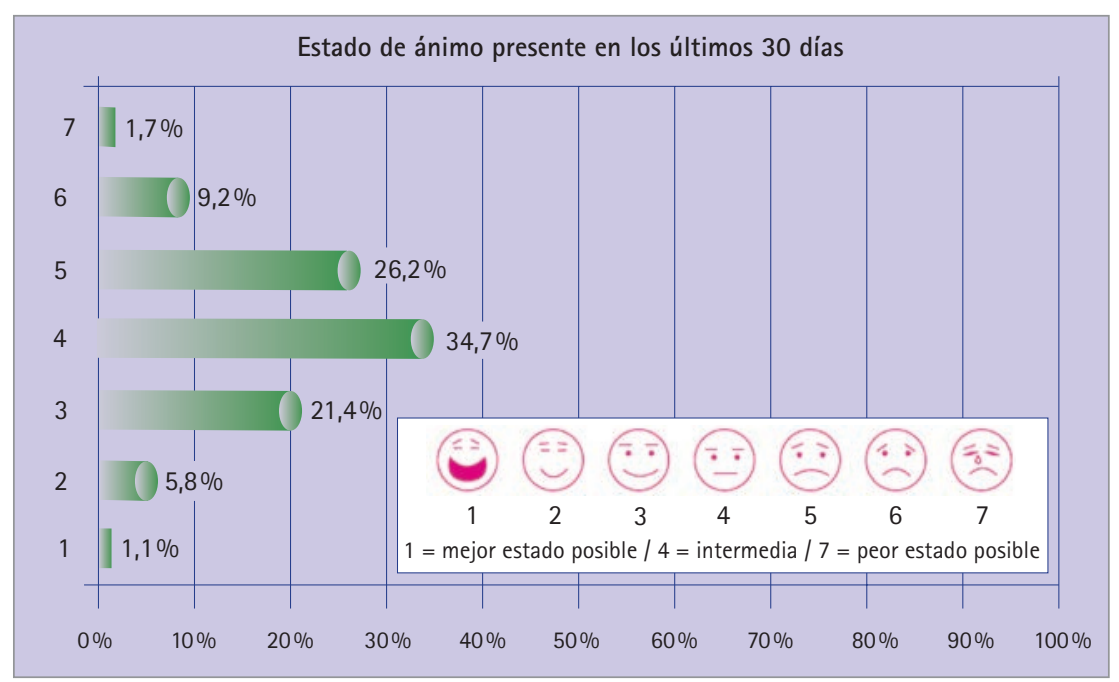

Figura 2 Estado de ánimo

gradación del 1 (risa) al 7 (llanto), el $34,7 \%$ de los sujetos manifestó una gradación 4 intermedia, siendo globalmente superior $(37,1 \%)$ el porcentaje de sujetos que manifestó algún grado de tristeza (5-7) e inferior (28,3\%) entre los que manifestaron algún grado de felicidad (1-3) (figura 2).

En relación al estado de salud, en la tabla 2 constan desglosados todos los ítems del cuestionario completo. En relación a la movilidad, el 36,2\% manifestó haber tenido algunos problemas para hacer actividades enérgicas, tales como correr, cargar objetos pesados, o participar en deportes activos. Respecto al cuidado personal el 91,7\% manifestó no haber tenido problema alguno para bañarse o vestirse solo. En cuanto a dolor y malestar, el 43,9\% manifestó haber tenido poco dolor de cuerpo o molestia, y al 34\% sí que el dolor le supuso cierta dificultad (escala: poco) para su trabajo habitual. Las habilidades interpersonales se afectaron sólo en el 38,2 \% de los sujetos, que manifestó que sólo alguna vez su salud física o los problemas emocionales le había dificultado sus relaciones personales o sus actividades sociales. En relación al sueño y energía, el 42,2\% manifestó que algunas veces tuvo problemas para quedarse dormido, el 37,7\% manifestó que algunas veces se sintió lleno de energía frente al 36,3\% que manifestó que solo alguna vez se sintió lleno de energía, y el 44,9\% algunas veces se sintió cansado.

En cuanto al ánimo, como sentimiento de tristeza, desánimo, preocupación y 
Tabla 2 Estado de salud

\begin{tabular}{|c|c|c|c|c|c|}
\hline Dimensiones & \multicolumn{5}{|c|}{ Valoración, n (\%) } \\
\hline Movilidad & Ninguno & Algunos & Bastantes & Muchos & $\begin{array}{c}\text { No lo podia } \\
\text { hacer }\end{array}$ \\
\hline $\begin{array}{l}\text { ¿Ha tenido problemas para ponerse de pie después de estar } \\
\text { sentado? }\end{array}$ & $\begin{array}{c}1054 \\
(69,7)\end{array}$ & $\begin{array}{c}354 \\
(23,4)\end{array}$ & $\begin{array}{c}72 \\
(4,8)\end{array}$ & $\begin{array}{c}30 \\
(2,0)\end{array}$ & $\begin{array}{c}2 \\
(0,1)\end{array}$ \\
\hline ¿Ha tenido problemas para moverse de un lado a otro? & $\begin{array}{c}1160 \\
(76,8)\end{array}$ & $\begin{array}{c}276 \\
(18,3)\end{array}$ & $\begin{array}{c}56 \\
(3,7)\end{array}$ & $\begin{array}{c}17 \\
(1,1)\end{array}$ & $\begin{array}{c}2 \\
(0,1)\end{array}$ \\
\hline $\begin{array}{l}\text { ¿Ha tenido problemas para subir varios escalones o para } \\
\text { caminar cuesta arriba? }\end{array}$ & $\begin{array}{c}909 \\
(60,5)\end{array}$ & $\begin{array}{c}426 \\
(28,3)\end{array}$ & $\begin{array}{r}119 \\
(7,9)\end{array}$ & $\begin{array}{c}44 \\
(2,9)\end{array}$ & $\begin{array}{c}5 \\
(0,3)\end{array}$ \\
\hline $\begin{array}{l}\text { ¿Ha tenido problemas para hacer actividades enérgicas, tales } \\
\text { como correr, cargar objetos pesados, o participar en deportes } \\
\text { activos? }\end{array}$ & $\begin{array}{c}536 \\
(35,6)\end{array}$ & $\begin{array}{c}545 \\
(36,2)\end{array}$ & $\begin{array}{c}253 \\
(16,8)\end{array}$ & $\begin{array}{l}120 \\
(8,0)\end{array}$ & $\begin{array}{c}53 \\
(3,5)\end{array}$ \\
\hline Cuidado personal & Ninguno & Algunos & Bastantes & Muchos & $\begin{array}{c}\text { No lo podia } \\
\text { hacer }\end{array}$ \\
\hline ¿Ha tenido problemas para bañarse o vestirse solo? & $\begin{array}{l}1385 \\
(91,7)\end{array}$ & $\begin{array}{c}99 \\
(6,6)\end{array}$ & $\begin{array}{c}19 \\
(1,3)\end{array}$ & $\begin{array}{c}5 \\
(0,3)\end{array}$ & $\begin{array}{c}2 \\
(0,1)\end{array}$ \\
\hline Dolor y malestar & Ninguno & Poco & Bastantes & Mucho & Extremo \\
\hline ¿Cuánto dolor de cuerpo o molestia ha tenido? & $\begin{array}{c}381 \\
(25,2)\end{array}$ & $\begin{array}{c}664 \\
(43,9)\end{array}$ & $\begin{array}{c}357 \\
(23,6)\end{array}$ & $\begin{array}{c}103 \\
(6,8)\end{array}$ & $\begin{array}{c}6 \\
(0,4)\end{array}$ \\
\hline $\begin{array}{l}\text { ¿Hasta qué punto el dolor le ha dificultado su trabajo } \\
\text { habitual (incluyendo las tareas domésticas y el trabajo fuera } \\
\text { de casa)? }\end{array}$ & $\begin{array}{c}644 \\
(42,6)\end{array}$ & $\begin{array}{c}513 \\
(34,0)\end{array}$ & $\begin{array}{c}258 \\
(17,1)\end{array}$ & $\begin{array}{c}86 \\
(5,7)\end{array}$ & $\begin{array}{c}9 \\
(0,6)\end{array}$ \\
\hline Habilidades interpersonales & $\begin{array}{l}\text { En ningún } \\
\text { momento }\end{array}$ & Sólo alguna vez & Algunas veces & Casi siempre & Siempre \\
\hline ¿Ha tenido problemas para bañarse o vestirse solo? & $\begin{array}{c}272 \\
(18,0)\end{array}$ & $\begin{array}{c}577 \\
(38,2)\end{array}$ & $\begin{array}{c}532 \\
(35,2)\end{array}$ & $\begin{array}{r}119 \\
(7,9)\end{array}$ & $\begin{array}{c}11 \\
(0,7)\end{array}$ \\
\hline Sueño y energía & $\begin{array}{l}\text { En ningún } \\
\text { momento }\end{array}$ & Sólo alguna vez & Algunas veces & Casi siempre & Siempre \\
\hline $\begin{array}{l}\text { ¿Con qué frecuencia tuvo problemas para quedarse dormido, } \\
\text { porque se despertaba varias veces durante la noche, o } \\
\text { porque se despertaba demasiado temprano en la mañana? }\end{array}$ & $\begin{array}{c}153 \\
(10,1)\end{array}$ & $\begin{array}{c}296 \\
(19,6)\end{array}$ & $\begin{array}{c}637 \\
(42,2)\end{array}$ & $\begin{array}{c}366 \\
(24,2)\end{array}$ & $\begin{array}{c}59 \\
(3,9)\end{array}$ \\
\hline ¿Cuánto tiempo se sintió lleno de energía? & $\begin{array}{l}143 \\
(9,5)\end{array}$ & $\begin{array}{c}547 \\
(36,3)\end{array}$ & $\begin{array}{c}568 \\
(37,7)\end{array}$ & $\begin{array}{c}225 \\
(14,9)\end{array}$ & $\begin{array}{c}23 \\
(1,5)\end{array}$ \\
\hline ¿Con qué frecuencia se sintió cansado? & $\begin{array}{c}35 \\
(2,3)\end{array}$ & $\begin{array}{c}224 \\
(15,0)\end{array}$ & $\begin{array}{c}672 \\
(44,9)\end{array}$ & $\begin{array}{c}491 \\
(32,8)\end{array}$ & $\begin{array}{c}75 \\
(5,0)\end{array}$ \\
\hline Comprensión, concentración e interacción & Ninguno & Algunos & Bastantes & Muchos & $\begin{array}{c}\text { No lo podia } \\
\text { hacer }\end{array}$ \\
\hline $\begin{array}{l}\text { ¿Ha tenido problemas para concentrarse o hacer algo } \\
\text { durante } 10 \text { minutos? }\end{array}$ & $\begin{array}{c}549 \\
(36,4)\end{array}$ & $\begin{array}{c}637 \\
(42,2)\end{array}$ & $\begin{array}{c}233 \\
(15,4)\end{array}$ & $\begin{array}{c}87 \\
(5,8)\end{array}$ & $\begin{array}{c}4 \\
(0,3)\end{array}$ \\
\hline $\begin{array}{l}\text { ¿Ha tenido problemas para recordar cosas importantes por } \\
\text { hacer? }\end{array}$ & $\begin{array}{c}491 \\
(32,6)\end{array}$ & $\begin{array}{c}662 \\
(43,9)\end{array}$ & $\begin{array}{c}263 \\
(17,5)\end{array}$ & $\begin{array}{c}85 \\
(5,6)\end{array}$ & $\begin{array}{c}6 \\
(0,4)\end{array}$ \\
\hline $\begin{array}{l}\text { ¿Ha tenido problemas para analizar y solucionar problemas } \\
\text { de la vida diaria? }\end{array}$ & $\begin{array}{c}578 \\
(38,4)\end{array}$ & $\begin{array}{c}663 \\
(44,0)\end{array}$ & $\begin{array}{c}190 \\
(12,6)\end{array}$ & $\begin{array}{c}72 \\
(4,8)\end{array}$ & $\begin{array}{c}3 \\
(0,2)\end{array}$ \\
\hline
\end{tabular}




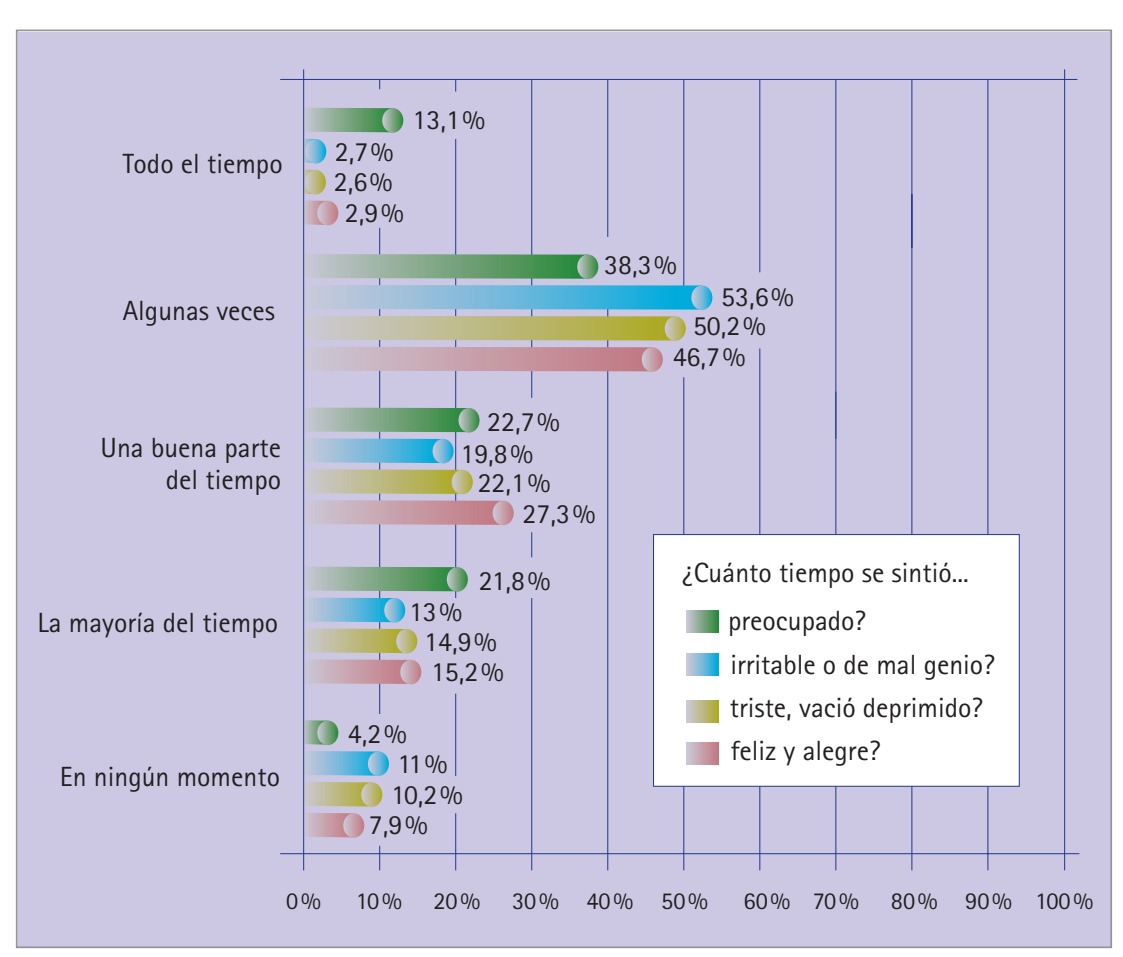

Figura 3 Ánimo

ansiedad (figura 3), la respuesta mayoritaria a todas las preguntas fue algunas veces: el 46,7\% de los sujetos se sintió feliz y alegre, el 50,2\% se sintió triste, vacío y deprimido, el 53,6\% se sintió irritable o de mal genio, y el 38,3\% se sintió preocupado.

En relación al ítem comprensión, concentración e interacción (tabla 2), el 42,2\% de sujetos manifestó haber tenido algunos problemas para concentrarse o hacer algo durante $10 \mathrm{mi}$ nutos, el 43,9\% manifestó haber tenido algunos problemas para recordar cosas importantes por hacer, y el 44,0\% manifestó haber tenido algunos problemas para analizar y solucionar problemas de la vida diaria.

Debido a la salud física, el 61,2\% manifestó que hizo menos de lo que hubiera querido hacer y el 44,9\% que tuvo que dejar de hacer algunas tareas en su trabajo o en sus actividades cotidianas. Respecto a si habían tenido problemas en el trabajo o actividades cotidianas a causa de algún problema emocional, el $66,2 \%$ manifestó que hizo menos de lo que hubiera querido hacer y el 54,0\% que tuvo que dejar de hacer algunas tareas en su trabajo o en sus actividades cotidianas.

$\mathrm{Al}$ analizar la importancia relativa entre problemas emocionales y salud física, se observó que existía una relación estadísticamente significativa relativo a los sujetos que hacen menos o dejan de hacer tareas cotidianas o las hacen de forma menos cuidadosa que de costumbre $(p<0,001)$.

\section{Estado de ánimo deprimido} sulta, el 61,8\% de los sujetos había consultado por un estado de ánimo deprimido, que en un 38,1\% de sujetos habían indicado estado de ánimo deprimido así como ansioso, nervioso e irritable.

Finalmente, el 12,0\% de sujetos había indicado que durante la mayor parte del día y durante más de dos semanas presentaba estado de ánimo deprimido, pérdida de interés y fatiga (falta de energía).

\section{Discusión}

Por lo que sabemos, este es el primer estudio realizado en nuestro país para conocer el perfil de los sujetos que cumplen criterios diagnósticos de trastorno adaptativo que acuden al farmacéutico. Los farmacéuticos pueden detectar situaciones en las que las personas manifiestan encontrarse en un estado de ánimo caracterizado por fatiga y deterioro en las actividades cotidianas, a las que les cuesta trabajo enfrentarse y demanden ayuda a su farmacéutico, solicitando consejo y apoyo para esta situación.

Los trastornos adaptativos presentan aparentemente una elevada
De acuerdo con el motivo de con- prevalencia, aunque hay pocos estudios al respecto, especialmente en atención primaria y psiquiatría de interconsulta donde la prevalencia oscila entre un 11-18\% y entre un $10-35 \%$ respectivamente $(9,10)$. En España, Gómez-Macías y col (11) encontraron una incidencia estimada del 13,5\% en un centro de salud, analizando pacientes nuevos con este diagnóstico en un periodo de 8 meses. No obstante, aunque el DSM-IVTR señala que el trastorno adaptativo es un diagnóstico común, no ha sido incluido en ninguno de los grandes estudios de epidemiología psiquiátrica (12-16), y hasta el momento sólo uno de los grandes estudios epidemiológicos llevados a cabo en la población general lo ha incluido entre las categorías de trastorno con sintomatología depresiva examinadas; el proyecto ODIN (Outcome of Depression International Network Project) $(17,18)$, que además tuvo en consideración únicamente el subtipo con estado de ánimo deprimido. Una razón para la escasa atención científica que ha recibido el trastorno adaptativo, a pesar de su elevada presencia en el ámbito clínico, es la inestabilidad que se atribuye al diagnóstico de este trastorno, así como que haya estado subordinado a otros trastornos, particularmente los del estado de ánimo.

En el presente estudio, el mayor porcentaje de mujeres con sintomatología de trastorno adaptativo que de hombres concuerda con los resultados hallados en otro estudio donde se determinó que ser mujer predice de forma significativa la presencia de depresión subclínica (19). En cuanto el estado civil, no concuerda en nuestro estudio que el estar viudo se asocie de forma significativa con un peor estado de salud, el mayor porcentaje corresponde a los casados seguido de los solteros (19).

En relación a la educación, en la muestra de sujetos analizada en este artículo, un 38,1\% de ellos tenía un nivel de estudios superiores universitarios y un 36,2\% tenía estudios secundarios finalizados. A este respecto, un análisis reciente ha puesto de manifiesto que una proporción significativa de los estudiantes universitarios de primer año experimentaban un conjunto de síntomas de adaptación que cumplían los criterios de un trastorno adaptativo, mientras que otra parte de ellos experimentaban 
también dichos sintomas pero a nivel subclínico (20).

Por otra parte, las situaciones estresantes como la pérdida de trabajo, cambios vitales, etc., constituyen un factor desencadenante del trastorno adaptativo (3), siendo en nuestro estudio importante destacar el elevado porcentaje de sujetos activos laboralmente $(62,2 \%)$ con trastornos adaptativos, siendo los principales factores estresores manifestados las dificultades económicas $(39,9 \%)$ y los problemas familiares $(32,9 \%)$.

Entre los sujetos del presente estudio, se observó que los síntomas motivo de la consulta tenían un impacto claramente negativo sobre su calidad de vida, con una elevada prevalencia de fatiga/falta de energía (78,2\%), estado de ánimo deprimido (61,8\%), ansiedad, nerviosismo y/o irritabilidad $(59,7 \%)$, pérdida de interés por las cosas con las que habitualmente disfrutaba (54,5\%), etc. Más del 75\% de estos sujetos presentaban un tiempo de evolución de los síntomas, la mayor parte del día y durante más de dos semanas.

En el caso de la depresión subclínica, las prevalencias encontradas son inferiores a las de los trastornos adaptativos en general, así la prevalencia de la depresión subclínica oscila entre el 2,2-24\% entre la población general, y entre 5-16\% entre los pacientes de atención primaria (21). La depresión subclínica se asoció con malestar psicológico significativo, discapacidad y pobre percepción de la salud (21). Los sujetos con depresión subclínica sufren un mayor impacto en su calidad de vida que los sujetos sin síntomas depresivos, siendo los sujetos con depresión mayor los que tienen el peor impacto en su calidad de vida (22). En el caso concreto del estado de ánimo de los sujetos objeto de estudio, hubo un mayor porcentaje de sujetos que manifestaron algún grado de tristeza $(37,1 \%)$ que de sujetos que manifestaron algún grado de felicidad $(28,3 \%)$, con un 50,2\% de los sujetos que se sintió triste, vacío y deprimido algunas veces, un 53,6\% que se sintió irritable o de mal genio algunas veces y un $38,3 \%$ que se sintió preocupado algunas veces. Por otra parte, alrededor del $40 \%$ de los sujetos manifestaron que algunas veces habian tenido problemas relacionados con el sueño y la energía, así como algunos proble- mas de comprensión, concentración e interacción. En los resultados destaca globalmente que más del 50\% de los sujetos manifestaron haber tenido problemas en el trabajo o actividades cotidianas a causa de algún problema emocional, encontrándose una relación estadísticamente significativa entre problemas en el trabajo o actividades cotidianas y problemas emocionales y salud física.

En relación con la correcta detección y tratamiento de los síntomas depresivos, existen diversas publicaciones sobre detección y tratamiento en la consulta de atención primaria que demuestran la existencia de depresión no detectada e infratratada (23-25). Aunque los médicos no psiquiatras parecen ser más eficaces para descartar correctamente alteraciones depresivas que para reconocerlas adecuadamente, particularmente en lo que atañe a los sintomas subclínicos de la depresión, un estudio reciente basado en el análisis de 660 historias clínicas de pacientes con síntomas subclínicos determinó que los médicos de atención primaria habían realizado un diagnóstico correcto de trastorno depresivo (26).

Aparte del tratamiento farmacológico, existen diversos tratamientos alternativos que son habitualmente utilizados por las personas con depresión $\mathrm{u}$ otras alteraciones psiquiátricas, siendo los más habitualmente utilizados la terapia de luz, acupuntura, yoga, suplementos dietéticos y de plantas medicinales, etc. Una encuesta realizada en pacientes de atención primaria puso de manifiesto que alrededor del $11 \%$ de las personas que sufrían depresión y ansiedad recurrían a terapias alternativas (27). Existen evidencias del beneficio de algunos tratamientos alternativos para el tratamiento de trastornos depresivos, que justifican el interés por este tipo de terapias y la elaboración de guías al respecto durante las últimas décadas que han sido objeto de revisión reciente (28).

Debido a que los trastornos afectivos se han relacionado con un bajo funcionamiento serotoninérgico, el papel de la serotonina (5-HT) sobre el estado de ánimo con o sin ansiedad ha sido objeto de numerosos estudios a partir de cuyos resultados se espera que el triptófano pueda mejorar el estado de ánimo en poblaciones vulnerables (29-32). En estos casos, son frecuentes asociarlos a problemas relacionados con el insomnio.

Los resultados de este estudio muestran el perfil del sujeto que acude a la farmacia comunitaria. También se pone de manifiesto el papel que pueden tener los farmacéuticos para poder ayudar a detectar precozmente y a mejorar el cuidado de los sujetos con trastornos adaptativos, gracias a la accesibilidad del farmacéutico que puede detectar señales de alerta, situaciones de riesgo e incluso efectos colaterales asociados a la medicación. De esta manera el farmacéutico podría ayudar a individuos presumiblemente afectados por un trastorno adaptativo y derivarlo a su médico para su valoración.

Así pues, a partir de los resultados de este estudio se puede concluir sobre el perfil de los sujetos que refieren sintomatología de trastornos adaptativos en la farmacia comunitaria que los principales motivos de consulta fueron en el 78,2\% de los sujetos fatiga/falta de energía, y en el 61,8\% estado de ánimo deprimido y el 59,7\% ansiedad, nerviosismo e irritación. Siendo síntomas de frecuente consulta, se podría sugerir que los farmacéuticos con su valoración farmacéutica podrían ayudar en la detección precoz de la posible presencia de trastornos adaptativos en la población, y a poder lograr con su intervención farmacéutica que las personas con elevado riesgo de depresión recibieran la atención necesaria para gestionar su condición.

\section{Agradecimientos}

A todos los farmacéuticos que participaron en la recogida de datos para el Estudio Energy, a Esther Pellicer por la preparación del manuscrito, y a Dynamic por su trabajo con el análisis estadístico.

\section{Referencias bibliográficas}

1. American Psychiatric Association. Diagnostic and Statistical Manual of Mental Disorders, Fourth Edition, Text Revision (DSM-IV-TR). Washington, DC: American Psychiatric Association; 2000.

2. Casey P, Bailey S. Adjustment disorders: the state of the art. World Psychiatry. 2011;10:11-18.

3. Strain JJ, Smith GC, Hammer JS, McKenzie DP, Blumenfield M, Muskin $P$, et al. Adjustment disorder: a multisite study of its utilization and 
interventions in the consultationliaison psychiatry setting. Gen Hosp Psychiatry 1998 May;20(3):139-149. doi:10.1016/S0163-8343(98)00020-6

4. Despland JN, Monod L, Ferrero F. Clinical relevance of adjustment disorder in DSM-III-4 and DSM-IV. Compr Psychiatry 1995;36:454-460. doi:10.1016/S0010-440X(95)90254-6

5. European Medicines Agency (EMEA). Note for guidance on Good Clinical Practice CPMP/ICH/135/95. [Internet]. July 2002. [acceso 12/1/2014]. Disponible en: http://ethikkommission. meduniwien.ac.at/fileadmin/ethik/ media/dokumente/rechtsgrundlagen/ GCP.pdf

6. Jiménez-Morón D, Saiz-Ruiz J. Una escala facial de depresión para poblaciones de bajo nivel cultural: resultados preliminares en una muestra de pacientes psiquiátricos ambulatorios. Psiquiatria.com [revista electrónica] 1998 Marzo; 2(1):[17 pantallas]. Disponible en: URL: http://www.psiquiatria. com/psiquiatria/vol2num1/art_5.htm

7. Ware JE, Kosinski M, Keller SD. A 12-Item Short-Form Health Survey. Construction of scales and preliminary tests of reliability and validity. Med Care. 1996;34:220-33. doi:10.1097/00005650-19960300000003

8. Badia X, Roset M, Montserrat S, Herdman M, Segura A. The Spanish version of EuroQoL: a description and its applications. European Quality of Life scale. Med Clin (Barc) 1999;112 (Supl 1):79-85.

9. Blacker CV, Clare AW. The prevalence and treatment of depression in general practice. Psychopharmacology (Berl) 1988;95(Suppl):S14-S17. doi:10.1007/ BF00172624

10. Silverstone PH. Prevalence of psychiatric disorders in medical inpatients. J Nerv Ment Dis 1996;184:43-51. doi:10.1097/00005053-19960100000008

11. Gómez Macías V, de Cós Milas A, García Blanco C, García Sánchez F, Ballesteros García M, Jiménez Ávalos MC. Trastornos adaptativos: factores estresantes en el momento actual. [Internet]. Comunicación presentada en: $7^{\circ}$ Congreso Virtual de Psiquiatría. Interpsiquis 2006. Psiquiatria.com. Febrero de 2006. [acceso 16/2/2014]. Disponible en http://hdl.handle. net/10401/3839

12. Helzer JE, Robins LN, McEvoy L. Post-traumatic stress disorder in the general population. Findings of the epidemiologic catchment area survey. N Engl J Med 1987;317:1630-1634.
doi:10.1056/NEJM198712243172604

13. Jenkins R, Bebbington $P$, Brugha $T$, Farrell M, Gill B, Lewis G, et al. The National Psychiatric Morbidity Surveys of Great Britain-strategy and methods. Int Rev Psychiatry 2003;15:5-13. doi:10.1080/0954026021000045895

14. Jenkins R, Lewis G, Bebbington $P$, Brugha T, Farrel M, Gill B, Meltzer H. The National Psychiatric Morbidity Surveys of Great Britain-initial findings from the household survey. Int Rev Psychiatry 2003;15:29-42. doi:10.1080/0954026021000045921

15. Kessler RC, Sonnega A, Bromet E, Hughes M, Nelson CB. Posttraumatic stress disorder in the National Comorbidity Survey. Arch Gen Psychiatry 1995;52:1048-1060. doi:10.1001/ archpsyc.1995.03950240066012

16. Breslau N, Kessler RC, Chilcoat HD, Schultz LR, Davis GC, Andreski P. Trauma and posttraumatic stress disorder in the community: the 1996 Detroit Area Survey of Trauma. Arch Gen Psychiatry 1998;55:626-632. doi:10.1001/archpsyc.55.7.626

17. Ayuso-Mateos JL, Vázquez-Barquero JL, Dowrick C, Lehtinen V, Dalgard OS, Casey P, et al. Depressive disorders in Europe: prevalence figures from the ODIN study. Br J Psychiatry 2001;179:308-316. doi:10.1192/ bjp.179.4.308

18. Dowrick C, Casey P, Dalgard 0, Hosman C, Lehtinen V, Vázquez-Barquero JL, et al. Outcomes of Depression International Network (ODIN). Background, methods and field trials. ODIN Group. Br J Psychiatry 1998;172:359363. doi:10.1192/bjp.172.4.359

19. Rivas M, Nuevo R, Ayuso-Mateos JL. Depresión subclínica en España: prevalencia e impacto sobre la salud. Rev Psiquiatr Salud Ment (Barc.) 2011;4:144-149. doi:10.1016/j. rpsm.2011.05.005

20. Rodgers LS, Tennison LR. A preliminary assessment of adjustment disorder among first-year college students. Arch Psychiatr Nurs 2009;23(3):220-230. doi:10.1016/j. apnu.2008.05.007

21. Rucci P, Gherardi S, Tansella M, Piccinelli M, Berardi D, Bisoffi G, et al. Subthreshold psychiatric disorders in primary care: prevalence and associated characteristics. J Affect Disord 2003;76:171-181. doi:10.1016/S01650327(02)00087-3

22. Da Silva Lima AF, de Almeida Fleck MP. Subsyndromal depression: an impact on quality of life? J Affect Disord 2007;100:163-169. doi:10.1016/j. jad.2006.10.010
23. Lecrubier Y. Widespread underrecognition and undertreatment of anxiety and mood disorders: results from $3 \mathrm{Eu}-$ ropean studies. The Journal of Clinical Psychiatry 2007;68(Supl 2):6-41.

24. Cameron IM, Lawton K, Reid IC. Appropriateness of antidepressant prescribing: an observational study in a Scottish primary-care setting. The British Journal of General Practice 2009;59:644-649. doi:10.3399/bjgp09X454061

25. Henriksson S, Asplund R, Boethius G, Hällström T, Isacsson G. Infrequent use of antidepressants indepressed individuals (an interview and prescription database study in a defined Swedish population 2001-2002). European Psychiatry: The Journal of the Association of European Psychiatrists 2006;21:355-360. doi:10.1016/j. eurpsy.2006.04.003

26. Cameron IM, Lawton K, Reid IC. Recognition and subsequent treatment of patients with sub-threshold symptoms of depression in primary care. J Affect Disord 2011;130:99-105. doi:10.1016/j.jad.2010.10.010

27. Roy-Byrne PP, Bystritsky A, Russo J, Craske MG, Sherbourne CD, Stein MB. Use of herbal medicine in primary care patients with mood and anxiety disorders. Psychosomatics 2005;46:117-122. doi:10.1176/appi.psy.46.2.117

28. Ravindran AV, Lam RW, Filteau MJ, Lespérace F, Kennedy SH, Parikh SV, et al. Canadian Network for Mood and Anxiety Treatments (CANMAT) Clinical guidelines for the management of major depressive disorder in adults. V. Complementary and alternative medicine treatments. J Affect Disord 2009; 117(Supl 1):S54-S64.

29. Silber BY, Schmitt JAJ. Effects of tryptophan loading on human cognition, mood, and sleep. Neurosci Biobehav Rev 2010;34:387-407. doi:10.1016/j. neubiorev.2009.08.005

30. Pearson NJ, Johnson LL, Nahin RL. Insomnia, trouble sleeping, and complementary and alternative medicine: analysis of the 2002 national health interview survey data. Arch Intern Med 2006;166:1775-1782. doi:10.1001/archinte.166.16.1775

31. Kessler RC, Chiu WT, Demler O, Merikangas KR, Walters EE. Prevalence, severity, and comorbidity of 12-month DSM-IV disorders in the National Comorbidity Survey Replication. Arch Gen Psychiatry 2005;62:617-627. doi:10.1001/archpsyc.62.6.617

32. Sarris J, Byrne GJ. A systematic review of insomnia and complementary medicine. Sleep Med Rev. 2011;15:99106. doi:10.1016/j.smrv.2010.04.001 


\section{Anexo. Guía del estudio}

\section{SELECCIÓN DE LOS SUJETOS}

\section{ADULTO QUE ACUDE A LA FARMACIA SOLICITANDO AYUDA Y CONSEJO}

Presenta alguno/s de los siguientes sintomas:

"Estado de ánimo bajo", desgana, cansancio, inapetencia, desmotivación, agobio, estrés o insomnio

La persona utiliza expresiones para definir su estade como: "estoy decaido', "duermo mal","no duermo yno me puedo levantar","me levanto cansado","me faltan las fuerzas, "estoy a pagado" 6 "estoy sobrepasado"

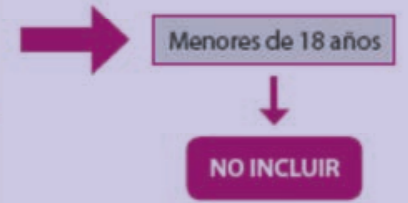

¿Ha habido algûn episodio e situación estresante en los últimos meses que puede ser la causa de ese estado de ánimo?

Episodio o situación estresante: pérdida de trabajo, problemas económicos, ruptura de una relación sentimental/separación/divorcio, problemas familiares, jubilación, problemas relacionados con la enseńanza, otros problemas ambientales o sociales, etc.

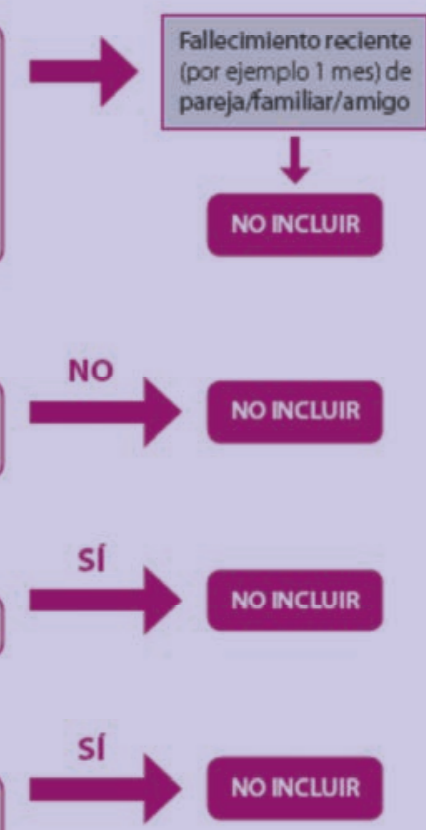

¿Tiene en la actualidad una depresión diagnosticada u otro tipo de trastorno psicológico o psiquiátrico?

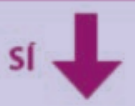

¿Su estado de ánimo está afectando su capacidad para continuar trabajando, en sus actividades cotidianas, sus relaciones sociales y personales?

$$
\text { si }
$$

¿Han pasado más de 6 meses desde el episodio o situación estresante?

\section{NO}

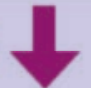

NO

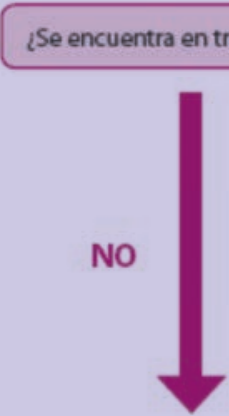

¿Los tratamientos que toma el paciente tienen asociados efectos secundarios que se corresponden con los sintomas que describe el paciente?

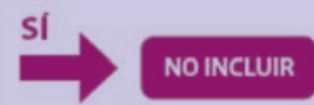

EL SUJETO CUMPLE TODOS LOS CRITERIOS PARA SER INCLUIDO EN EL ESTUDIO 


\section{OBTENCION DEL CONSENTIMIENTO INFORMADO DE LOS SUJETOS}

- Al tratarse de una investigación que recoge datos personales y médicos del sujeto, el estudio debe presentarse a un Comité Ético de Investigación Clínica (CEIC), que comprobará que cumple con la normativa ética actual, respetando la confidencialidad y anonimato de los sujetos en todo momento. Así mismo, se ha informado del estudio a la Agencia Española del Medicamento y Productos Sanitarios (AEMPS).

Es imprescindlble pedir la autorización del sujeto para la recogida y posterior utilización de sus datos personales y médicos.

- Una vez que haya confirmado que el paciente cumple los criterios de selección para participar en este estudio, antes de recoger ninqún dato debe obtener su autorización por escrito mediante la firma de un consentimiento informade

- Para que el sujeto pueda decidir si quiere o no participar en el estudio, usted debe facilitarle un documento llamado "Hoja de Información al Paciente" donde se le informa de aspectos del estudio para pueda decidir si quiere participar en el mismo. En dicho documento se explica de forma sencilla los siguientes aspectos fundamentales:

Objetivo del estudio: recogida de datos de una población que presenta síntomas de desánimo, desmotivación, cansancio, agobio, estrés o duerme mal, y que acude a la farmacia pidiendo ayuda para este problema. Esta población es muy habitual en las farmacias, pero hay muy poca información al respecto.

\section{Participación en el estudio:}

* La participación del sujeto es absolutamente voluntaria.

* La participación consistirá únicamente en una pequeña entrevista que usted le realizará, y en la que recogerá una serie de datos sociales y demográficos (edad, situación civil, situación laboral, etc), se le preguntará sobre las enfermedades que padece, sobre su estado de ánimo y de salud.

\section{Confidencialidad:}

* El estudio cumple con lo estipulado en la Ley de Protección de Datos vigente en España.

* Toda la información que sea recogida será confidencial y anónima.

》El sujeto estará Identificado únicamente con un código numérico que será asignado según el orden de entrada en el estudio

- El sujeto debe saber que tanto usted como sus colaboradores han firmado un compromiso de confidencialldad y que tienen el deber de no revelar sus datos en ningún momento si no es con su consentimiento.

* Los datos serán analizados junto con los datos de los otros participantes y podrán ser usados para presentaciones o publicaciones cientificas siempre respetando el anonimato de los sujetos. 
En todos los casos, se seguirá siempre el siguiente orden de procedimientos antes de la recogida de datos:

Comprobación de los criterios de selección para poder incluir al sujeto (Criterios de inclusión/exclusión)

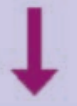

Informar sobre el estudio y facilitar la "Hoja de Información al paciente" al sujeto

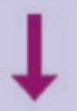

Firma del "Formulario de Consentimiento Informado" $\longrightarrow$ Registrar la fecha de la firma en el CRD

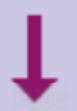

Recogida de datos en el Cuaderno de Recogida de Datos 


\section{RECOGIDA DE DATOS EN EL CUADERNO DE RECOGIDA DE DATOS}

Puntuaciones e interpretación de la escala de estado de ánimo y el cuestionario de estado de salud

\section{Evaluación del estado de ánimo (escala de caras)}

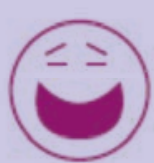

Mejor estado posible

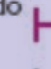

1

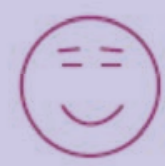

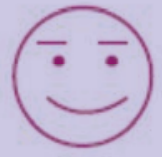
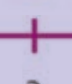
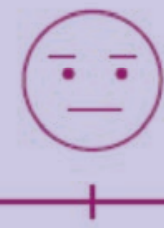

4
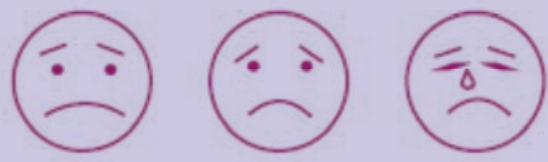

Peor estado posible

Se valorará el estado de ánimo del paciente con una escala de caras dónde " 1 " es el "mejor estado de ánimo posible" $\mathrm{y}^{\text {" }} 7^{\circ}$ el "peor estado de ánimo posible", pasando por diferentes posibilidades de estado de ánimo intermedias.

\section{Evaluación del estado de salud (cuestionario)}

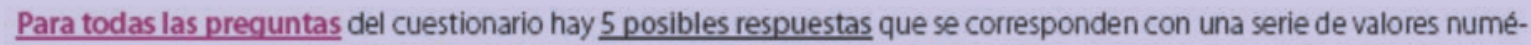
ricos, y cuánto más alta es la puntuación peor será la situación del sujeto:

- "1" es el "mejor estado posible"

- " "5" es el "peor estado de ánimo posible"

Movilidad

Cuidado personal

Comprensión, concentración e interacción

\begin{tabular}{|c|c|c|c|c|c|c|}
\hline \multirow{3}{*}{$\begin{array}{l}\text { Mejor estado } \\
\text { posible }\end{array}$} & guno & Algunos & Bastantes & Muchos & $\begin{array}{l}\text { No lo podía } \\
\text { hacer }\end{array}$ & \\
\hline & $F$ & + & + & + & -1 & \multirow[t]{2}{*}{$\begin{array}{r}\text { Peor estado } \\
\text { posible }\end{array}$} \\
\hline & 1 & 2 & 3 & 4 & 5 & \\
\hline
\end{tabular}




\section{Dolory malestar}

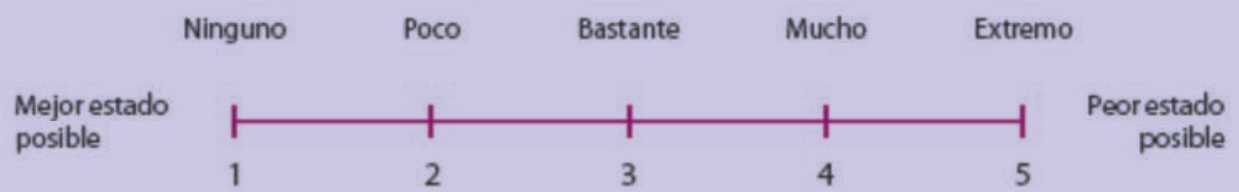

Habilidades interpersonales

Sueño y energía (preguntas 9 y 11)

\begin{tabular}{|c|c|c|c|c|c|c|}
\hline & $\begin{array}{l}\text { En ningún } \\
\text { momento }\end{array}$ & $\begin{array}{c}\text { Sólo alguna } \\
\text { vez }\end{array}$ & $\begin{array}{c}\text { Algunas } \\
\text { veces }\end{array}$ & Casi siempre & Siempre & \\
\hline \multirow{2}{*}{$\begin{array}{l}\text { Mejor estado } \\
\text { posible }\end{array}$} & F & + & + & + & -1 & \multirow{2}{*}{$\begin{array}{r}\text { Peor estado } \\
\text { posible }\end{array}$} \\
\hline & 1 & 2 & 3 & 4 & 5 & \\
\hline
\end{tabular}

Excepción: en el caso de la pregunta 10 de la dimensión de "sueño y energia", y la pregunta 12 de la dimensión de "ánimo", uánto más alta es la puntuación, mejor será la situación del sujeto.

\section{Sueño y energía (pregunta 10)}

\begin{tabular}{|c|c|c|c|c|c|c|}
\hline \multirow{3}{*}{$\begin{array}{l}\text { Peor estado } \\
\text { posible }\end{array}$} & $\begin{array}{l}\text { En ningún } \\
\text { momento }\end{array}$ & $\begin{array}{c}\text { Sollo alguna } \\
\text { vez }\end{array}$ & $\begin{array}{c}\text { Algunas } \\
\text { veces }\end{array}$ & Casi siempre & Siempre & \multirow{3}{*}{$\begin{array}{r}\text { Mejor estado } \\
\text { posible }\end{array}$} \\
\hline & 5 & + & + & + & -1 & \\
\hline & 1 & 2 & 3 & 4 & 5 & \\
\hline
\end{tabular}

Ánimo (pregunta 12)

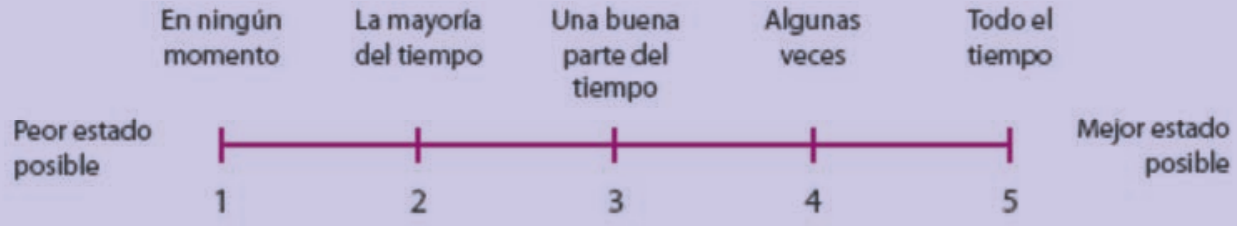

